\title{
A mini review on thiadiazole compounds and their pharmacological interest
}

\author{
Mohammad Asif ${ }^{1, *}$, Abida $^{2}$ \\ ${ }^{\mathbf{1}}$ Associate Professor, ${ }^{2}$ Assistant Professor, ${ }^{1}$ Dept. of Pharmacy, ${ }^{2}$ Dept. of Pharmaceutical Chemistry, ${ }^{\mathbf{1}}$ Guru Ram Das (PG) Institute of \\ Management and Technology, Dehradun, Uttarakhand, India, ${ }^{2}$ Faculty of Pharmacy, Northern Border University, Rafha, 91911 , PO Box \\ 840, Saudi Arabia
}

\section{*Corresponding Author: Mohammad Asif}

Email: aasif321@gmail.com

\begin{abstract}
Various 1,3,4-thiazole derivatives have been reported to exhibit various biological activities. The 1,3,4-thiadiazole derivatives found to have diverse pharmacological activities such as, insecticidal, herbicidal, antiviral, anti-tumor, CNS stimulant, anti-bacterial, antifungal, antiangiogenic, antiglaucoma, antiischemic, and anti-inflammatory, antidepressant, anxiolytic, antiparasitic, antitumor, hypoglycemic, antihypertensive and CNS depressant activities The 1,3,4-Thiadiazoles have also been used in many fields and majority of applications as dyes, lubricants, analytical reagents and agents. The 1,3,4-Thiadiazole analogs are associated with diverse biological activities probably by virtue of toxophoric - $\mathrm{N}=\mathrm{C}-\mathrm{S}$ - group. Due to the wide range of applications we have studied thiadiazole derivatives for their biological significances.
\end{abstract}

Keywords: Thiadiazoles, Pharmacological, Triazolo.

\section{Introduction}

Thiadiazole derivatives are well known for their significant biological activities. A large number of thiadiazoles have been reported to exhibit various biological activities. Some 1,3,4-thiadiazole derivatives found to have diverse pharmacological activities such as, insecticidal, herbicidal, anti-tumor, antifungal, antiangiogenic, antiglaucoma, antiischemic, and anti-inflammatory, antidepressant [HI], anxiolytic, anti-bacterial, antiparasitic, antitumor, hypoglycemic antihypertensive, antiviral, anti HIV, anti-HSVl, antiproteolytic, antiphage, antithyroidal, antiamoebic, anticonvulsant, neurotoxicity, insecticidal, herbicidal and also plant growth regulators, CNS depressant and CNS stimulant properties. ${ }^{1-7}$ Many of them have potential biological usage and some have been tried for antituberculosis. They also find applications as dyes, lubricants, analytical reagents and antiviral agents. The 1,3,4-Thiadiazole analogs are associated with diverse biological activities probably by virtue of toxophoric $-\mathrm{N}=\mathrm{C}-$ $\mathrm{S}$ - group. The 1,3,4-Thiadiazoles have applications in many fields and majority of applications are patented. Some of the thiadiazole analogue act as nematicides and cefazolin (5methyl-1,3,4-thiadiazole-2-thiol derivative, is used as an antibacterial agent. ${ }^{7-10}$

The 1,3,4-Thiadiazole analogues have displayed activity against hepatitis B virus. 2-Amino-1,3,4-thiadiazole5 -thiol is an effective radioprotective agent. The most pronounced effect of amino thiadiazole on ribonucleotide pools of leukemia L 1210 cells is the lowering of guanine ribonucleotide pools. They act as potent orally active nonpeptide antagonist for the bradykinin $\mathrm{p} 2$ receptor and induce mortality in frog embryos. A series of thiosemi-carbazides and investigated them as central cholecystokinin and neurokinin receptors. Thiosemicarbazides are also considered as psychotropic agents. The most important industrial uses of thiosemicarbazides are they act as corrosion inhibitors of copper, and carbon-steel, in aqueous chloride solutions. Also they act as thermal stabilizers for rigid poly vinyl chloride. ${ }^{11-15}$ The thiadiazole drugs were the first effective chemotherapeutic agents to be employed systematically for the prevention and cure of bacterial infection in human beings (eg: Sulphamethizole).They are also choice for the drug as diuretic (eg: Acetazolamide). Thiadiazole derivatives are well known to have number of biological and antimicrobial, anti-inflammatory and anticonvulsant activities.

Chemistry: Thiadiazole is a heterocyclic compound featuring both two nitrogen atom and one sulphur atom as part of the aromatic five-membered ring. Thiadiazole and related compounds are called 1,3,4-thiadiazole (two nitrogen and one other heteroatom in a five membered ring). They occur in nature in four isomeric forms as. 1,2,3thiadiazole; 1,2,5-thiadiazole; 1,2,4-thiadiazole and 1,3,4thiadiazole.

Biological importance 1,3,4-Thaidiazoles: The Heterocyclic nucleus 1,3,4-thiadiazole constitutes an important class of compounds for new drug development. The synthesis of thiadiazole derivatives for their chemical and biological behaviors and have gained more importance in recent decades. In recent years there has been intense investigation of different classes of thiadiazole compounds, many of which possess extensive pharmacological activities.

Various thiadiazole have been shown to possess different activities. The fungicidally active PyrazolylSubstituted-1,3,4-thiadiazole compounds, the preliminary bioassay tests indicated that compounds (1) and (2) have fungicidal activity. ${ }^{16}$ A series of 2sulfonamido/trifluoromethyl-6- substituted imidazo [2,1-b]1,3,4-thiadiazole derivatives (3). The selected compounds were evaluated for their preliminary in vitro antituberculosis activity against Mycobacterium tuberculosis. Some of the compounds exhibited moderate to good antitubercular activity. ${ }^{17}$ 
<smiles>CCc1nn(C)c(-c2n[nH]c(=S)s2)c1Cl</smiles>

1

Antibacterial activity of $N$-(5-benzylthio-1,3,4thiadiazol-2-yl) and $N$-(5-benzylsulfonyl-1,3,4-thiadiazol-2yl)piperazinyl quinolone derivatives (4) against Grampositive and Gram-negative microorganisms). Some of these derivatives exhibit high activity against Gram positive bacteria Staphylococcus aureus and S. epidermidis,<smiles>[R]c1cccc(-c2nc3sc([R])nn3c2[R])c1</smiles>

$3 \mathbf{R}_{\mathbf{1}}=-\mathrm{SO}_{2} \mathrm{NH}_{2},-\left(\mathrm{CH}_{3}\right)_{2} \mathrm{~N}-\mathrm{C}=\mathrm{N}-\mathrm{SO}_{2}-\mathrm{CH}_{3}$, $\mathrm{CF}_{3} \quad \mathbf{R}_{2}=\mathrm{CH}_{3}, \mathrm{Cl}$, OMe, Trimethoxy, $\mathrm{NO}_{2}$ $\mathbf{R}_{3}=\mathrm{H},-\mathrm{SCN},-\mathrm{C}=\mathrm{N}-\mathrm{NH}-\mathrm{C}=\mathrm{N}$ H-NH${ }_{2}$

comparable or more potent than their parent $N$-piperazinyl quinolones norfloxacin and Ciprofloxacin as reference drugs. ${ }^{18} \quad \mathrm{~A}$ set of $\mathrm{N}$-substituted 2-amino5-(2,4dihydroxyphenyl)-1,3,4-thaidaizole derivatives. Among these compound (5) showed a very good anticancer and neuroprotective activity. ${ }^{19}$<smiles>[R][R]1cccc(CSc2nnc(N3CCN(c4cc5c(cc4F)c(=O)c(C(=O)O)cn5[R])CC3)s2)c1</smiles>

$\mathbf{4} \mathbf{R}=$ ethyl, cyclopropyl $\mathbf{R}_{1}=\mathrm{H}, \mathrm{NO}_{2}$<smiles>Oc1ccc(-c2nnc(Nc3ccc(F)cc3)s2)c(O)c1</smiles>

A series of selective cox-2 inhibitors with 2-amino-5sulfanyl-1,3,4-thiadiazole derivatives (6) were selective inhibitiors of COX-2 and potentiated the activity of COX-1 enzyme. The presence of sulphonamide group is a required

pharmacophore for selective inhibition of COX-2 enzyme. ${ }^{20}$ The syntheses of various compounds were evaluated for antidiabetic activity. Among of these compounds (7) have shown significant antidiabetic activity. ${ }^{21}$

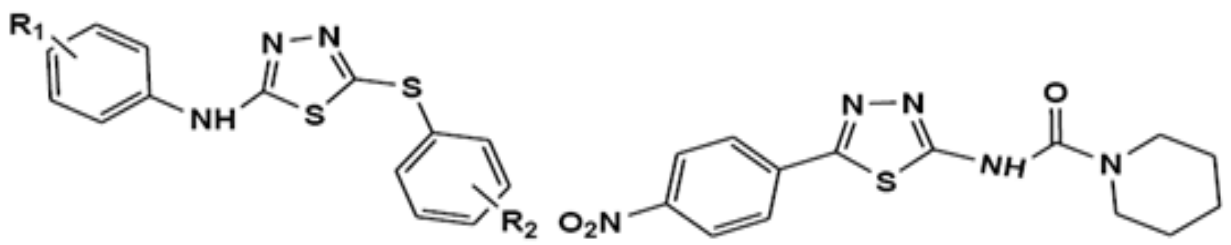

$6 \mathrm{R}_{1}=\mathrm{F}, \mathrm{CH}_{3}, \mathrm{CF}_{3}, \mathrm{SO}_{2} \mathrm{NH}_{2} \mathrm{R}_{2}=\mathrm{SO}_{2} \mathrm{NH}_{2}, 7$

The 1,3,4-thiadiazole, that containing a phenylalanine moiety were synthesized by intra-molecular cyclization of 1,4-thiosmicrbazides (8), in acid and alkaline media and the synthesized compounds was evaluated by anti-inflammatory activity. ${ }^{22}$ The 2-(arylmethanesulfonylmethyl)-5-aryl-1,3,4thiadiazoles (9) exhibited high activity on both Gram (+ve) and Gram (-ve) bacteria. ${ }^{23}$

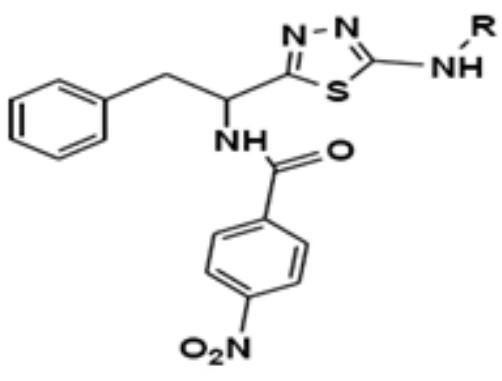

8

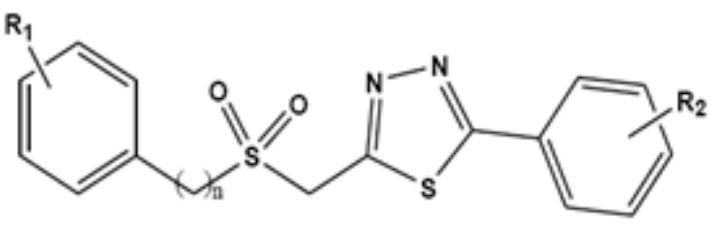

9
The investigation to identify selective antagonists, and they found that aminothiadiazole that is compound (10) was identified from a high throughput screen as having good antagonist activity for human EP3. ${ }^{24}$ Derivatives of 2,4- 
Substituted diphenyl-5-imino- $\Delta^{2}$-1,3,4-thiadiazole (11) were evaluated their antimicrobial properties. These compounds exhibited promising antimicrobial activity. ${ }^{25}$<smiles>CCC(CC)c1nnc(NC(=O)c2ccc3c(c2)OCCO3)s1</smiles>

10

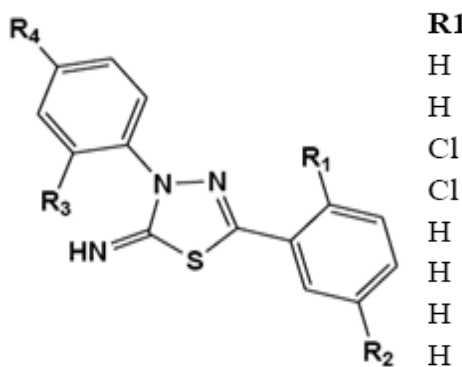

11
A series of 1,3,4-thaidaizoles, the synthesized compounds were screened for their in vitro antibacterial activity. All the newly synthesized compounds (12) were initially screened for their in vitro antibacterial activities against the Gram-positive ( $S$. aureus, S. cerevisiae and $C$. diphtheriae) and the Gram negative (E.coli and $P$. aeruginosa) bacteria by agar cup-plate method not disc diffusion method. ${ }^{26} \mathrm{~A}$ series of 2-amino-5-aryl-thiazolo $[1,3,4]$-Thiadiazole derivatives (13) were prepared. Some of the synthesized compounds showed very good antitubercular activity. ${ }^{27}$<smiles>Nc1nnc(CSc2nnc(SCc3nnc([Tl])s3)s2)s1</smiles><smiles>[R]C1SC=C2SC(N)=NN21</smiles>

$13 \mathrm{R}=\mathrm{Ph}, 4-\mathrm{MeC}_{6} \mathrm{H}_{4}, 4-\mathrm{OHC}_{6} \mathrm{H}_{4},-\mathrm{NO}_{2} \mathrm{C}_{6} \mathrm{H}_{4}, 4 \mathrm{Me}_{2} \mathrm{NC}_{6} \mathrm{H}_{4}, 2 \mathrm{ClC}_{6} \mathrm{H}_{4}$, $4-\mathrm{ClC}_{6} \mathrm{H}_{4}, 2,4-\mathrm{Cl}_{2} \mathrm{C}_{6} \mathrm{H}_{3}, 2 \mathrm{OMeC}_{6} \mathrm{H}_{4}, 3-\mathrm{OMeC}_{6} \mathrm{H}_{4}$

The 2,4-Thiazolidinediones bearing Imidazo[2,1b] $[1,3,4]$ Thiadiazole derivatives (14-16) show very good antimicrobial activity. ${ }^{28}$<smiles>[R]c1ccc(-c2cn3nc(S(N)(=O)=O)sc3n2)cc1</smiles>

14

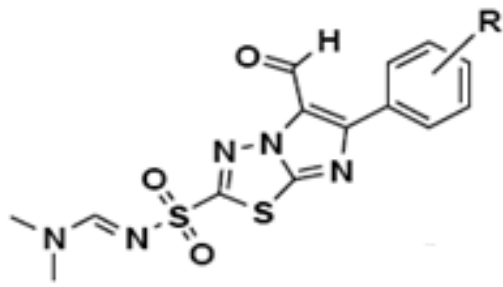

15<smiles>[R]c1ccc(-c2nc3sc(S(=O)(=O)N=CN(C)C)nn3c2/C=C2\SC(=O)NC2=O)cc1</smiles>

16

$\mathbf{R}=\mathrm{H}, 4-\mathrm{Br}, 4-\mathrm{Cl}, 2,5-(\mathrm{OMe}) 2,4-\mathrm{CH}_{3}, 4-\mathrm{OMe}, 4-\mathrm{NO}_{2}$

A series of 3-(1,3,4-Thiadiazole-2-yl) quinoline derivatives (17) from chloroquinone with an aim to explore their effect on in vitro growth of microorganisms causing microbial infection. ${ }^{29}$ The 6-aryl-2-(2-aryl-2H-1,2,3-triazol4 -yl) imidazo[2,1-b]-1,3,4- thiadiazoles (18). Some of these compounds were found to possess slight to moderate activity against the microorganisms Staphylococcus aureus, Candida albicans, Pseudomonas aeruginosa, and Escherichia coli. ${ }^{30}$ 
<smiles>[X]c1cccc2cc(C3SC(N([R])C(C)=O)=NN3C(C)=O)c(Cl)nc12</smiles>

$17 \mathbf{X}=\mathrm{H}, \quad \mathrm{Cl}, \mathrm{CH}_{3} \mathbf{R}=\mathrm{H}$, Cyclohexyl, O-tolyl, 4nitrophenyl, cyclopentyl, 2,5-difluoro phenyl,

A series of 2-cyclopropyl Imidazo [2,1-b] [1,3,4]Thiadiazole derivatives, among the compounds tested, compound $(19,20)$ found to be the most active candidate of<smiles>[R][R]1cccc(-c2nc3sc(-c4cnn(-c5ccc[R]([R])c5)n4)nn3c2I)c1</smiles>

18

the series at five dose level screening with degree of selectivity towards Leukemic cancer cell line. ${ }^{31}$

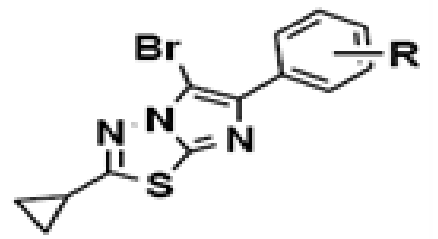

$19 \mathbf{R}=\mathrm{H}, 4-\mathrm{Cl}$, 4-Br, 4-F, 2,4-Di-Cl, 2,4-di-OH, 3$\mathrm{NH}_{2}, 4-\mathrm{NH}_{2}, 3-\mathrm{NO}_{2}, 4-\mathrm{NO}_{2}$,

A series of novel Imidazo [2,1-b][1,3,4]-thiadiazole carrying rhodanine-3-acetic acid as potential antitubercular agents (21). Among synthesized compounds, some of the compounds showed very good vitro antitubercular activity

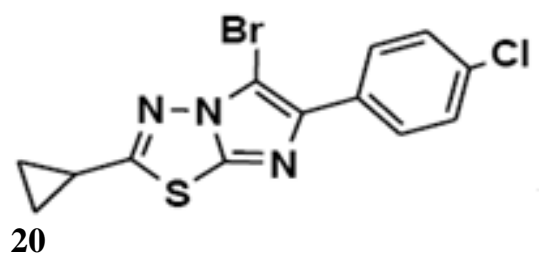

against M. tuberculosis. ${ }^{32}$ The phenytoin derivatives and studied its anticonvulsant activity. Among the synthesized compounds, only phenyl substituted (22) showed promising anticonvulsant activity. ${ }^{33}$

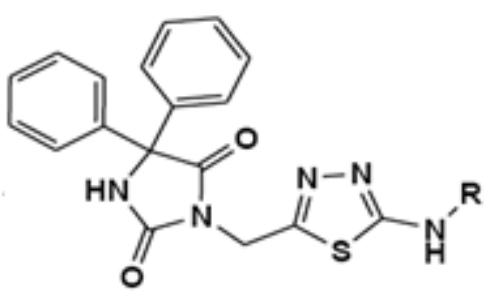

$22 \mathrm{R}=\mathrm{C}_{2} \mathrm{H}_{5}, \mathrm{C}_{6} \mathrm{H}_{5}, 4-\mathrm{CH}_{3} \mathrm{C}_{6} \mathrm{H}_{4}, 4-\mathrm{OMe}-\mathrm{C}_{6} \mathrm{H}_{4}, 4-\mathrm{Cl}-\mathrm{C}_{6} \mathrm{H}_{4}$

(24) were exhibited Anti-microbial activity of ${ }^{35}$ Synthesis, characterization and anticancer activity of 1,2,4-Triazolo [3,4-b]-1,3,4-thiadiazoles (25) on Hep G2 cell lines. ${ }^{36}$ antimicrobial; anti-inflammatory and antidiabetic agents. Fluoro benzothiazole incorporated with 1,3,4-Thiadiazoles<smiles>[R]OC(=O)CNc1nnc(Br)s1</smiles><smiles>CCN(C)CC</smiles><smiles>Fc1ccc2nc(NNc3nnc(-c4ccccc4Nc4ccccc4)s3)sc2c1Nc1ccccc1</smiles>

$24 \mathrm{R}=\mathrm{o}, \mathrm{m}, \mathrm{p}-$ methoxy<smiles>[R]c1nnc2sc(-c3c[nH]nc3Br)nn12</smiles>

$25 \mathrm{R}=$ Phenoxy methyl, $\mathrm{R} 1=\mathrm{p}-$ chloro phenyl 
Synthesis of pharmaceutically important 1,3,4thiadiazole and imidazolinone derivatives (26) as antimicrobials. $^{37}$ The 2,4-Di substituted-5-Imino-1,3,4Thiadiazole Derivatives (27) were exhibited antiinflammatory activities. ${ }^{38}$ Some Thiosemicarbazide and 1,3,4- Thiadiazole Heterocycles Bearing Benzo[b] Thiophene Nucleus (28) as a Potent Antitubercular and Antimicrobial Agents. ${ }^{39}$<smiles>[R]c1nnc(Nc2nc3ccccc3s2)s1</smiles>

$26 \mathrm{R}=$ Phenyl, 4-chloro phenyl, 4nitro phenyl<smiles>[R6]c1ccc([R8])c(-c2nn(-c3cc([R6])ccc3[R])c(=N)s2)c1</smiles>

$27 \mathrm{R}_{1}, \mathrm{R}_{2}, \mathrm{R}_{3}, \mathrm{R}_{4}=\mathrm{H}$<smiles>PNc1nnc(-c2sc3ccc(Oc4ccccc4)cc3c2Cl)s1</smiles>

$28 \mathrm{R}=$ Aryl
Synthesis of 2-Amino-5-sulfanyl-1,3,4-thiadiazoles (29), a series of selective cyclooxygenase-2 inhibitors. ${ }^{40}$
Evaluation of $\operatorname{Bis}[1,2,4]$ triazolo[3,4- $b][1,3,4]$ thiadiazoles (30) as Potent Antimicrobial Agents. ${ }^{41}$<smiles>[R]c1ccc(Sc2nnc(Nc3ccc(Br)cc3)s2)cc1</smiles>

$29 \mathrm{R}=\mathrm{SO}_{2} \mathrm{NH}_{2} \mathrm{R}_{1}=\mathrm{Cl}$

The Zn(II) Complex of Schiff Base Derived from 5Acetazolamido-1,3,4 -Thiadiazole-2-Sulphonamide (31), act as Diuretic Drug. ${ }^{42}$ Antimicrobial activity of 2-(2'substituted-benzylidene-hydrazino-acetyl)-mercapto-5-<smiles>CCc1nn2c(-c3cc(Cc4ccc(OC)c(-c5nnc6sc(Br)nn56)c4)ccc3OC)nnc2s1</smiles>

$30 \mathrm{Ar}=$ Phenyl, 2- chloro phenyl, 4-methyl phenyl

methyl-1,3,4-thiadiazoles (32) and 2-[2'-\{4-substituted-aryl3-chloro-2-oxo-azetidine -acetyl-amino-mercapto]-5methyl-1,3,4-thiadiazoles. ${ }^{43}$<smiles></smiles>

31

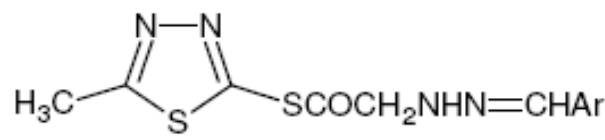

$32 \mathrm{Ar}=$ Substituted aryl groups

The aromatic aldehyde imine derivatives of 2anticonvulsant activity. ${ }^{44}$ Fungicidally Activity exhibited by thiobenzyl-1,3,4-thiadiazole (33) was exhibited Pyrazolyl-Substituted 1,3,4-Thiadiazole (34) Compounds. ${ }^{45}$

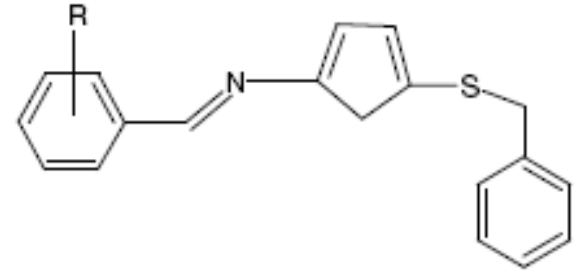

$33 \mathrm{R}=2-\mathrm{Cl}, 4-\mathrm{Cl}, 2-\mathrm{NO}_{2}, 4-\mathrm{NO}_{2}$<smiles>CCCc1nn(C)c(-c2nnc(SSc3nnc(-c4c(Cl)c(C)nn4C)s3)s2)c1Cl</smiles>

34 
The 1,3,4-Thiadiazole Derivatives of 5-Amino-2Hydroxybenzoic Acid (35) was exhibited Antimicrobial
Activity. ${ }^{46}$ Some 1,3,4-Thiadiazole Derivatives (36) were exhibited Antimicrobial Activity. ${ }^{47}$<smiles></smiles>

35<smiles>Cc1ccc([141I])cc1</smiles><smiles>Nc1nnc(CSc2nnc(SCc3nnc(N)s3)s2)s1</smiles>

36
The 3-[2-benzothiazolyl/benzimidazolyl methyl-1,2,4triazolo[3,4,b][1,3,4]thiadiazole-6-yl-subsituted (37) as possible anthelmintics. ${ }^{48}$ The 1,3,4-thiadiazole derivative (38) was exhibited fungicidal activity. ${ }^{49}$

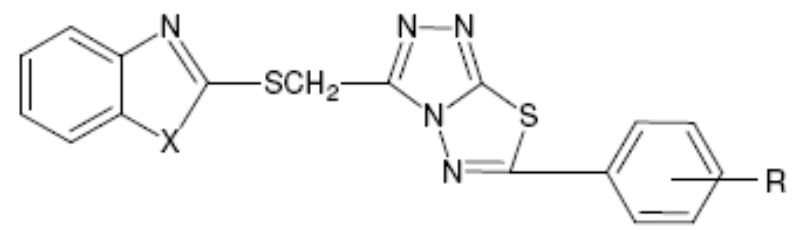

$37 \mathrm{X}=\mathrm{S}, \mathrm{O}$

The 1,3,4-thiadiazole derivative (39) were exhibited antinflamatory activity. ${ }^{50}$ The nicotinyl incorporated

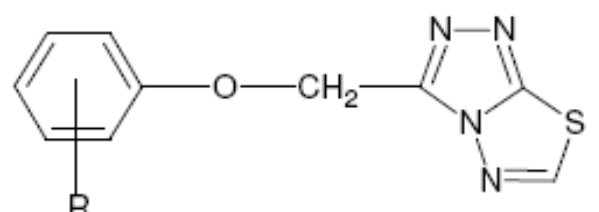

38

quinazolinonyl thiadiazoles (40) were exhibited possible NSAID activity. ${ }^{51}$<smiles>[R]CCCCNc1cccc(C(=O)N(c2nnc([R])s2)N2Cc3cc([X])cc([X])c3N=C2[R])c1</smiles>

40

The triazolo[3,4-b][1,3,4]thiadiazole (41) was exhibited antimicrobial activity. ${ }^{52}$ The 1,3,4-thiadiazole derivatives (42) were exhibited antimicrobial and anti-inflammatory

activities. ${ }^{53}$ The pyrazole, pyrazolones and oxadiazole bearing 2-arylamino-5-mercapto-1,3,4-thiadiazoles were exhibited antimicrobial activities. ${ }^{54}$

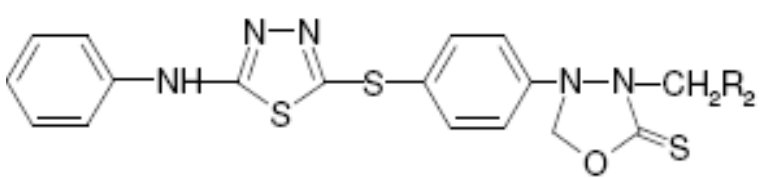

$43 \mathrm{R}_{1}=\mathrm{H}, \mathrm{CH}_{3}, \mathrm{Cl}$

$\mathrm{R}_{2}=$ Five membered Hectrocyclic moiety

41<smiles>C=C(C)c1nnc2sc(=S)[nH]n12</smiles>

42Ar $\mathrm{CH}_{2} \mathrm{OC}_{6} \mathrm{H}_{5} \mathrm{Cl}$ $=\mathrm{C}_{6} \mathrm{H}_{5} \mathrm{OCH}_{3}$,

The 3,5 disubstituted-s-triazolo (3,4-b)-1,3,4-thiadiazole (44) were exhibited antimicrobial and antinflamatory activities. ${ }^{55}$ The imidazolo[2,1-b]-1,3,4-thiadiazole (45) was exhibited antimicrobialactivity. ${ }^{56}$ 
<smiles>COc1ccc2cc(C(C)c3nn4c(Br)nnc4s3)ccc2c1</smiles>

44

The 1-(2-diazo-5-arylalkyl-1,3,4-thiadiazolyl)-6methoxy benzophenothiazines (46) were exhibited antiviral and antifungal activities. ${ }^{57}$ The 3-(2-thienyl)-s-triazolo[3,4-<smiles>[2H]c1ccc([N+](=O)[O-])c(-c2nn3c(Br)c(-c4ccc([N+](=O)[O-])cc4)nc3s2)c1</smiles>

45 b] $[1,3,4]$ thiadiazole (47) were exhibited antimicrobial and diuretic activities. ${ }^{58}$<smiles>[R]c1nnc(/N=N\c2c3c(cc4ccccc24)Sc2cc(OC)ccc2N3)s1</smiles>

46

The 2,5-disubstituted 1,3,4-thiadiazole derivatives (48) were exhibited antibacterial activity. ${ }^{59}$ The N-(1,3,4thiadiazole-2-yl)5-substituted-2-amino-4,5-disubstituted-<smiles>S=c1[nH]n2c(-c3cccs3)nnc2s1</smiles>

47<smiles>[R]c1cc2c(cc1[R])-c1nn(Cc3nncs3)c(=S)cc1CCC2</smiles>

48

Some 1,3,4-thiadiazole derivatives (50) were exhibited free radical scavenging activity. ${ }^{61}$ The aryl-N-(5-\{4-[2chlorophenyl)-4-oxo(3-hydroquinazoline)]-phenyl $\}(1,3,4-$ thiophen-3-carboxalic acid (49) exhibited analgesic and anti inflamatory agent. ${ }^{60}$<smiles>Nc1ccc(S(=O)(=O)Nc2nnc(S(N)(=O)=O)s2)cc1</smiles>

51<smiles>[R]c1nnc(Nc2sc([R2])c([R])c2C(=O)O)s1</smiles>

$$
49 \mathrm{R}=\mathrm{C}_{6} \mathrm{H}_{5} \mathrm{NH}, \mathrm{CH}_{3} \mathrm{NHCH}_{2} \mathrm{R}_{1}=\mathrm{R}_{2}=\mathrm{CH}_{3},\left(\mathrm{CH}_{2}\right)_{4}
$$

thiadiazol-2-yl)amides (51) were exhibited antibacterial and antifungal activities. ${ }^{62}$

Some 3-(5-aryl-1,3,4-thiadiazole-2-yl)-1-( $\beta$-Dglucopyra-nosyl)-5-alkyl-2-thio-4-imidazo-lidinones (53) were exhibited fungicidal activity. ${ }^{63}$<smiles>[R]c1nc2ccccc2c(=O)n1-c1ccc(-c2nnc(NC(=O)[Ga])s2)cc1</smiles>

52R= 2-chlorophenyl Ar=Different aryl groups 
<smiles>[R]c1ccc(-c2nnc(N3C(=O)C([R7])N(C4OC(CO)C(O)C(O)C4O)C3=S)s2)cc1</smiles>

\section{Conclusion}

The thiadiazole posses various pharmacological activities including anticancer, anti-inflammatory, antitubercular, antioxidant, antimicrobial, anticonvulasant, and analgesic activities, above observations promoted us to synthesize the new thiadiazole compounds with potent biological activities. ${ }^{64}$ The 1,3,4-thiadiazole are important because of their versatile biological actions. In particular, compounds bearing the 1,3,4-thiadiazole nucleus is known to have unique antibacterial and anti-inflammatory activities. Differently substituted thiadiazole moieties have also been found to have other interesting activities such as analgesic, antimicrobial, antitubercular, anticonvulsant and anti-hepatitis B viral and other useful activities.

\section{References}

1. Katritzky A R. Handbook of Heterocyclic Chemistry, Pergamon Press, New York, 1985.

2. Witkoaski J.T., Robins R.K., Sidwell R.W., Simon L.N., $J$ Med Chem 1972;15:150.

3. Katritzky A.R., Rees C.W., "Comprehensive Heterocyclic Chemistry: The structure, reaction, synthesis and uses of heterocyclic compounds" 1-edition. Potts, K.T. Pergamon press: Oxford: New York, 1984,6, 546.

4. Andreani A., Leoni A., Locatelli A., Morigi R., Rambaldi M., Simon W.A., J. Senn-Bilfinger, Arzneimittelforschung, 2000;50:550.

5. Clerici F., Pocar D., Guido M., Loche A., Perlini V., Brufani M., J Med Chem 2001;44:931.

6. Prasad R., Srivastava P.K. , Arch Pharm (Weinheim), 1993;326:963

7. Neelam B., Mannar M., Fehmida N., Alok B., Sudha B., Amir A., Eur J Med Chem 2000;35:481.

8. Yogeeswari P., Sriram D., Saraswat V., Ragavendran J.V., Kumar M.M., Murugesan S., Thirumurugan R., Stables J.P., Eur J Pharm Sci 2003;20:341.

9. Li J.P., Luo Q.F., Wang Y.L, Wang H., Synth Commun 2001;31:1793.

10. Karakus S., Rollas S., IL Farmaco, 2002;51:577.

11. Dziadulewicz E.K., Ritchie T.J., Hallett A., Snell C.R., Ko S.Y., Wrigglesworth R., Hughes G.A., Dunstan A.R., Bloomfield G.C., Drake G.S., Brown M.C., Lee W., Burgess G.M., Davis C, Yaqoob M., Perkins M.N., Campbell E.A., Davis A.J. and Rang H.P., J Med Chem 2000;43:769.

12. Mekenyan O.G., Schultz T.W., Veith G.D., Kamenska V., $J$ Appl Toxicol 996;16:355.

13. Dziadulewicz E.K., Walpole C.S., Snell C.R., Wrigglesworth R., Hughes G.A., Beattie D., Wood J.N., Beech M.M., Coote P.R., Bioorg Med Chem Lett 2001;11 705.
14. Singh M.M., Rastogi R.B., Upadhyay B.N., Yadav M., Mater Chem Phys 2003;80:283.

15. El-Shafei A.A., Moussa M.N.H., El-Far A.A., Mater Chem Phys, 2001;70:175.

16. Chen, H. S., Li, Z. M., Han, Y. F., Wang, Z. W. Chin. Chem Lett 1999;10(5):365-366.

17. Gadad, A. K., Noolvi, M. N., Karpoormath, R.V. Bioorg Med Chem 2004;12:5651-5659.

18. Foroumadi, A., Emami, S., Hassanzadeh, A., Rajaee, M., Sokhanvar, K., Moshafi, M. H., Shafiee, A. Bioorg Med Chem Lett 2005;5(20):4488-4492.

19. Rzeski, W., Matysiak, J., Szerszen, M. K. Bioorg. Med Chem 2007;15(9):3201-3207.

20. Sharma, R., Sainy, S., Chatuvedi, S. C. Acta Pharm 2008;58:317-326.

21. Pattan, S. R., Kekare, P., Dighe, N. S., Nirmal, S. A., Musmade, D. S., Parjane, S. K., Daithankar, A. V. J Chem Pharm Res 2009;1(1):191-198.

22. Moise, M., Sunel, V., Profire, L., Popa, M., Desbrieres, J., Peptu, C. Molecules 2009;14:2621-2631.

23. Padmavathi, V., Reddy, G. S., Padmaja, A., Kondaiah, P., AliShazia. Eur J Med Chem 2009;44:2106-2112.

24. Hilfiker, M. A., Wang, N., Xiaoping, H., Zhimin, D., Pullen, M. A., Nord, M., Nagilla, R., Fries, H. E., Wu Charlene, W., Sulpizio, A. C., Jaworski, J., Morrow, D., Edwards, R. M., Jian. Bio. Org. Med. Chem. Letters, 2009;19:4292-4295.

25. Asif M., Asthana, C. Acta Pharm. Sciencia, 2010;52:443-451.

26. Salimon, J., Salih, N., Hameed, A., Ibraheem, H., Yousif, E. J Appl Sci Res 2010;6(7):866-870.

27. Asif, K. A., Sunil, V. M., Prathap, K. J., Mukesh, S. K. Int Res J Pharm 2011;2(1):153-158.

28. Alagawadi, K. R., Alegaon, S. G. Arabian J Chem 2011;4:465472.

29. Bhat, A. R., Tazeem, Azam, A., Choi, I., Athar, F. Eur J Med Chem 2011;46:3158-3166.

30. Atta, K. F. M., Farahat, O. M., Ahmed, A. Z. A., Marei, M. G. Molecules 2011;16:5496-5506.

31. Alegaon, S. G., Kallanagouda, R. Pranali, A. V., Sagar, S. M., Chaudhary, Dilip H. D, Amol S. S. Bioorg Med Chem Lett 2012;22(5):1917-1921.

32. Yang, S. J., Lee, S. H., Kwak, H. J., Gong, Y. D. J Org Chem 2013;78:438-444

33. Botros, S., Khalil, N. A., Naguib, B. H., Dash, Y. E. Eur Med Chem 2013;60:57-63.

34. Gourely RN, Pat US. Oct. 4, 1977;4,052,379.

35. Pattan S. R, Kekare P, Dighe N.S, Nirmal S.A, Musmade D.S, Parjane S.K, Daithankar A.V. J Chem Pharm Res 2009;1(1):191-198.

36. Vedavathi M, Somashekar B, Sreenivasa G. M, Jayachandran E. J Pharm Sci Res 2010;2(1):53-63

37. Sunil D, Arun M Isloor and Prakash S. Der Pharma Chemica 2009;1(2):19-26.

38. Mohd Amir, Arun Kumar, Israr Ali \& Khan S. A. Ind J Chem 2009;48B: 1288-1293.

39. Mohammad Asif, Chhavi Asthana. Int J Chem Tech Res 2009;1(4):1200-1205.

40. Vasoya S.L, Paghdar D.J, Chovatia P.T, and Joshi H.S. J Sci Islam Republican Iran 2005;16(1):33-36.

41. Rajesh S, Jitendra S and Subhash C. C Acta Pharm 2008;58:317-326.

42. Cherkupally S. R, Lade S. R and Adki N. Acta Chim Slov 2010;57:726-732.

43. Suparna G, Suman M, Bharti J and Ganesh N. Asian J Exp Sci 2009;23(1):189-192.

44. Rajiv D and Srivastava S.K. Int J Pharma Bio Sci 2010;1(2),

45. Bahar A and Yusuf Md. Ind J Chem 2010;49(B):241-246.

46. Han Song Chen, Zheng Ming Li, Yu Feng Han, Zhong Wen Wang. Chinese Chem Lett, 1999;10(5):365-366. 
47. Sabir H, Jyoti S and Amir Mohd. E-J Chem 2008;5(4):963-968

48. Jumat S, Nadia S, Ayad H, Hiba I, Emad Y. J Appl Sci Res 2010;6(7):866-870.

49. Imtiaz Husain. M, Vinay Kumar, Ind J Chem 1992;31B:673676.

50. Bano. Q, Tiwari N, Nizamudin. Ind J Chem 1992;31B:714-718

51. Srinivasa. M, Rama Sharma G. V. S. Ind J Heterocyclic Chem 1998;7:281-284

52. Rama Sharma G.V.S, John Thomas, V. Murugan K. Elango J Heterocyclic Chem 1999;9:151-152.

53. Jag Mohan, Anupama. Ind J Chem 2001;40B:368-371.

54. Manjunath Ghate, D.Srinivasa. Ind J Heterocyclic Chem 2002;11:225-256.

55. Smita, Nair, S. P. Garg, Parimla Sah. Ind J Heterocyclic Chem 2002;12:09-12.

56. Udupi R. H, S. Ramachandra Setty, N. Srinivasulu, Nandkishore Agarwal, C.V. Suresh. Ind J Heterocyclic Chem 2002;12;33-36.

57. Jag Mohan, Ashok Kumar. Ind J Heterocyclic Chem 2002;12:41-44.
58. V.K.Pandey, H.S.Negi. Ind J Chem 2003;42B:206-210.

59. Jag Mohan. Ind J Chem 2003;42B:401-404.

60. Venkataswarlu Pesapati, Srikat Chitty. Ind J Chem 2003;42B:616-620.

61. Ishawar Singh, Rathod S, Patel D. J Heterocyclic Chem 2005; 14:281-284.

62. Chajed M.R., Khendekar P.B., Mund A.S. Ind J Heterocyclic Chem 2007;16:259-262.

63. Desai N.C, Shihora P.N, Moradia D.L. Ind J Chem 2007;46B:550-553.

64. Alok Kumar Srivastava, Khare R.R, Singh H. Ind J Chem 2007;46B: 875-879.

How to cite this article: Asif M, Abida. A mini review on thiadiazole compounds and their pharmacological interest. Int J Pharm Chem Anal 2018;5(4):156-164. 